University of Nebraska - Lincoln

DigitalCommons@University of Nebraska - Lincoln

Focusing of longitudinal ultrasonic waves in air with an aperiodic flat lens

John T. Welter

Air Force Research Laboratory, Nondestructive Evaluation Branch

Shamachary Sathish

University of Dayton Research Institute

Daniel Christensen

lowa State University

Philip Brodrick

Southwestern Ohio Council of Higher Education

Jason D. Heebl

Air Force Research Laboratory, Nondestructive Evaluation Branch

See next page for additional authors

Follow this and additional works at: https://digitalcommons.unl.edu/usafresearch

Part of the Aerospace Engineering Commons

Welter, John T.; Sathish, Shamachary; Christensen, Daniel; Brodrick, Philip; Heebl, Jason D.; and Cherry, Matthew, "Focusing of longitudinal ultrasonic waves in air with an aperiodic flat lens" (2011). U.S. Air Force Research. 35.

https://digitalcommons.unl.edu/usafresearch/35

This Article is brought to you for free and open access by the U.S. Department of Defense at DigitalCommons@University of Nebraska - Lincoln. It has been accepted for inclusion in U.S. Air Force Research by an authorized administrator of DigitalCommons@University of Nebraska - Lincoln. 


\section{Authors}

John T. Welter, Shamachary Sathish, Daniel Christensen, Philip Brodrick, Jason D. Heebl, and Matthew Cherry 


\title{
Focusing of longitudinal ultrasonic waves in air with an aperiodic flat lens
}

\author{
John T. Welter ${ }^{\text {a) }}$ \\ Air Force Research Laboratory, Nondestructive Evaluation Branch (AFRL/RXLP), 2230 Tenth Street, \\ Wright-Patterson AFB, Ohio 45433 \\ Shamachary Sathish \\ University of Dayton Research Institute, Structural Integrity Division, 300 College Park, Dayton, Ohio 45469 \\ Daniel E. Christensen \\ Iowa State University, Department of Aerospace Engineering, 12508 Crawford Road, Omaha, \\ Nebraska 68144 \\ Philip G. Brodrick \\ Southwestern Ohio Council of Higher Education, 7828 Shadowhill Way, Cincinnati, Ohio 45242 \\ Jason D. Heebl
Air Force Research Laboratory, Nondestructive Evaluation Branch (AFRL/RXLP), 2230 Tenth St.,
Wright-Patterson AFB, Ohio 45433 \\ Matthew R. Cherry \\ University of Dayton Research Institute, Structural Integrity Division, 300 College Park, Dayton, Ohio 45469
}

(Received 16 March 2011; revised 27 June 2011; accepted 12 August 2011)

\begin{abstract}
Modeling and experimental results of an ultrasonic aperiodic flat lens for use in air are presented. Predictive modeling of the lens is performed using a hybrid genetic-greedy algorithm constrained to a linear structure. The optimized design parameters are used to fabricate a lens. A method combining a fiber-disk arrangement and scanning laser vibrometer measurements is developed to characterize the acoustic field distribution generated by the lens. The focal spot size is determined to be 0.88 of the incident wavelength of $80-90 \mathrm{kHz}$ at a distance of $2.5 \mathrm{~mm}$ from the lens. Theoretically computed field distributions, optimized frequency of operation, and spatial resolution focal length are compared with experimental measurements. The differences between experimental measurements and the theoretical computations are analyzed. The theoretical calculation of the focal spot diameter is $1.7 \mathrm{~mm}$ which is $48 \%$ of the experimental measurement at a frequency of $80-90 \mathrm{kHz}$. This work illustrates the capabilities of a hybrid algorithm approach to design of flat acoustic lenses to operate in air with a resolution of greater than the incident wavelength and the challenges of characterizing acoustic field distribution in air. [DOI: 10.1121/1.3640841]
\end{abstract}

PACS number(s): 43.58.Ls, 43.20.Fn, 43.35.Yb, 43.20.Gp [AJZ]

Pages: $2789-2796$

\section{INTRODUCTION}

Typically focusing of acoustic waves has been achieved with lens structures based on refraction. Single element acoustic lenses based on refraction are an integral part of scanning acoustic microscopy (SAM). ${ }^{1}$ In SAM high frequency acoustic waves have been brought to focus with an acoustic lens on to the surface of a sample in presence of a coupling liquid. Single element acoustic lenses have been used to focus acoustic waves in presence of high pressure gases $^{2}$ and in ambient air. ${ }^{3}$ The focal spot size in single element lenses has been limited by diffraction and the spatial resolution, $s$, as determined by the Rayleigh criterion, $s=1.22(\lambda / D)$, where $\lambda$ is the wavelength of sound in the coupling medium and $D$ is the diameter of the lens. ${ }^{4}$ A single

\footnotetext{
a) Author to whom correspondence should be addressed. Electronic mail: John.Welter@wpafb.af.mil
}

element acoustic lens typically consists of a cylindrical rod with a piezoelectric transducer attached at one end. The opposite end has a spherical curvature that contacts the coupling liquid.

Planar acoustic lens structures based on Fresnel diffraction have been developed for focusing of acoustic waves also. ${ }^{5}$ The focal spot size and spatial resolution of Fresnel lenses have been determined by diffraction theory and the Rayleigh criterion. Although Fresnel lenses have planar structure, the individual corrugations may have thickness variations or steps for matching the phase of the acoustic field at the focal spot. Acoustic Fresnel lenses also consists of a cylindrical rod with corrugations on one end and a piezoelectric transducer at the opposite end. The corrugated structure is immersed in coupling fluid to focus acoustic waves on the sample surface. Both single element lenses and Fresnel lenses have been used to focus acoustic fields in the far field. 
In the last decade new concepts have been developed and used for lens designs. One of the designs has been based on the concept of negative refractive index..$^{6-11}$ These lenses have been demonstrated to have resolution better than diffraction limit. ${ }^{8}$ Following the negative refraction concept periodic grating structures have been explored for manipulation of electromagnetic and acoustic fields. ${ }^{12-16}$ The focusing in these new lenses and periodic grating structures has been based on near field diffraction theory. Although these structures have been demonstrated to have better resolution, they operated only in a very narrow frequency range and had serious practical limitations. Aperiodic grating structures have been explored for acoustic focusing also. ${ }^{17-21}$ An aperiodic grating structure has been optimized to achieve acoustic focusing in the near field through a combination of near field diffraction and multiple scattering theories. Initially the concept was demonstrated by focusing an acoustic beam to a line by optimally arranged cylindrical rods. ${ }^{16,17}$ Recently, Sanchis et al. has extended the approach to focus acoustic waves to circular spot by optimally arranging a ring structure with axial symmetry. ${ }^{22}$ This lens consisted of several rings of varying diameters arranged with the centers aligned along the line. The distance between rings, diameter of the rings and positioning of the rings was optimized using a genetic algorithm to operate at $2.2 \mathrm{kHz}$. Although the lens has a circular acoustic focal spot for acoustic imaging applications the structure was quite complicated.

This paper presents the design, simulation, and experimental demonstration of a circular structure to focus acoustic waves in air to a circular spot. The structure is designed using a genetic-greedy hybrid algorithm. The algorithm is used to determine optimized structural dimensions and theoretical focal spot size. Based on the optimized dimensions, the structure is fabricated from aluminum using electrical discharge machining (EDM). The structure is tested with an air coupled acoustic transducer and the focusing of the acoustic field is characterized by measuring the displacement of a $350 \mu \mathrm{m}$ diameter disk attached to a $50 \mu \mathrm{m}$ diameter fiber with a laser scanning vibrometer. The diameter of the focal spot was observed to be $3.52 \mathrm{~mm}$ at a distance of $2.5 \mathrm{~mm}$ from the lens for an acoustic frequency range of $80-90 \mathrm{kHz}$. The theoretical calculations of the focal spot diameter have been found to be $48 \%$ of the experimental measurements.

The paper first describes computational modeling and simulation of the circular structure to focus acoustic waves. The acoustic fields are computed and displayed along with the dimensions of the lens. In the next section the fabrication of the structure and the experimental measurements of acoustic fields are described. Finally, in the discussion section, the experimental results are compared with the theoretical computation and possible discrepancies are discussed.

\section{MODELING AND SIMULATION}

A schematic of the structure and acoustic focusing is shown in Fig. 1. Acoustic plane waves generated by a piezoelectric transducer are incident on the circular structure and focused to a point on the other side of the structure directly opposite to the transducer. Modeling the interaction of ultrasound with such a structure is a complex problem, and further modeling the resultant wave behavior after the interaction is also challenging. ${ }^{23}$ The complexity of these problems arise from parameters such as the number and type of boundaries involved, the number of reflections accounted for, the number and type of wave modes interacting, and how the focal spot is defined. For each dimension simulated this computational burden is multiplied. Several assumptions are made to make the model more manageable. It is first assumed that the smallest spatial resolution corresponds to highest degree of focusing, which is assumed to be at the point of maximum pressure. To further simplify the modeling a two-dimensional (2D) approach is used rather than the

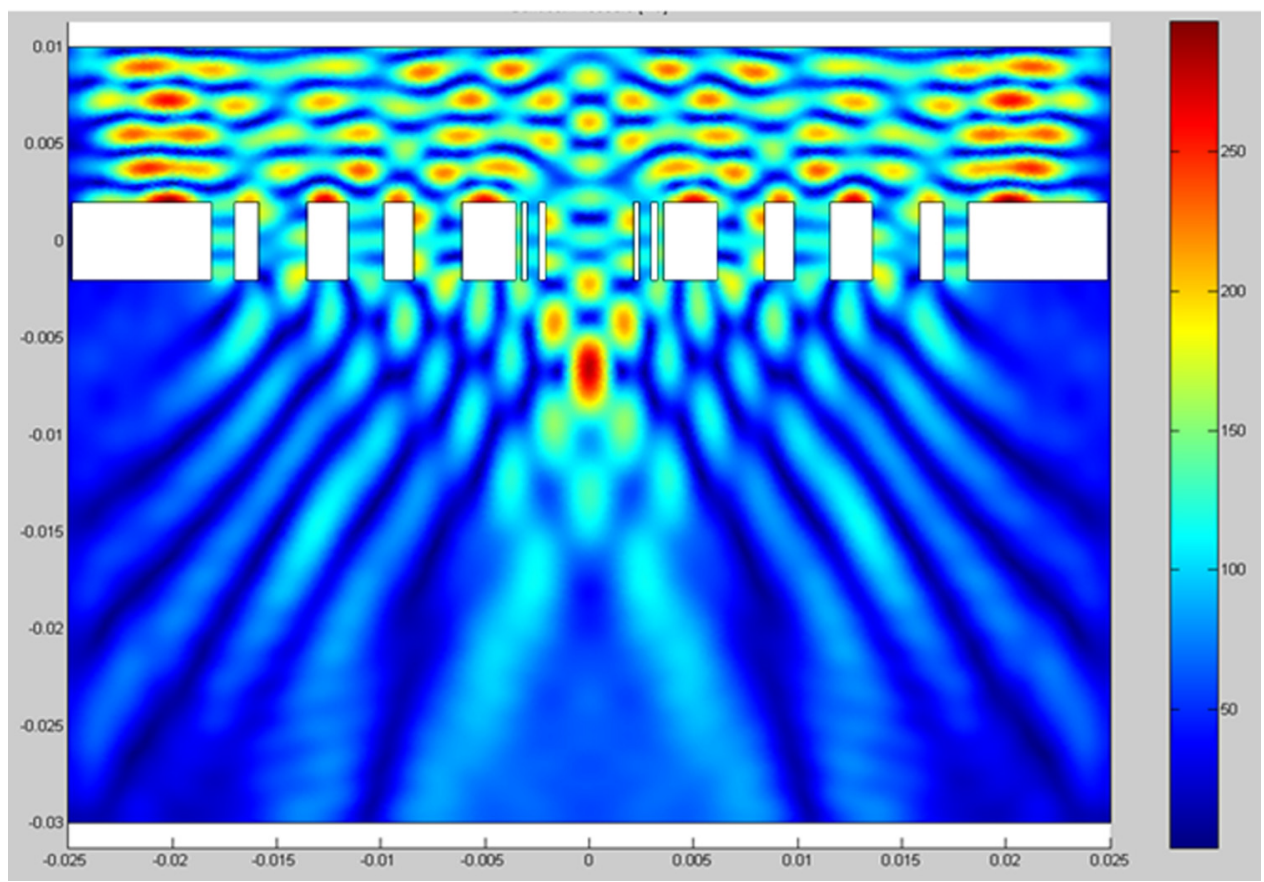

FIG. 1. (Color online) COMSOL simulation of the acoustic lens with a $100 \mathrm{kHz}$ incident wave. $X$ and $Y$ axes are position in meters. Color scale is pressure in arbitrary units. 


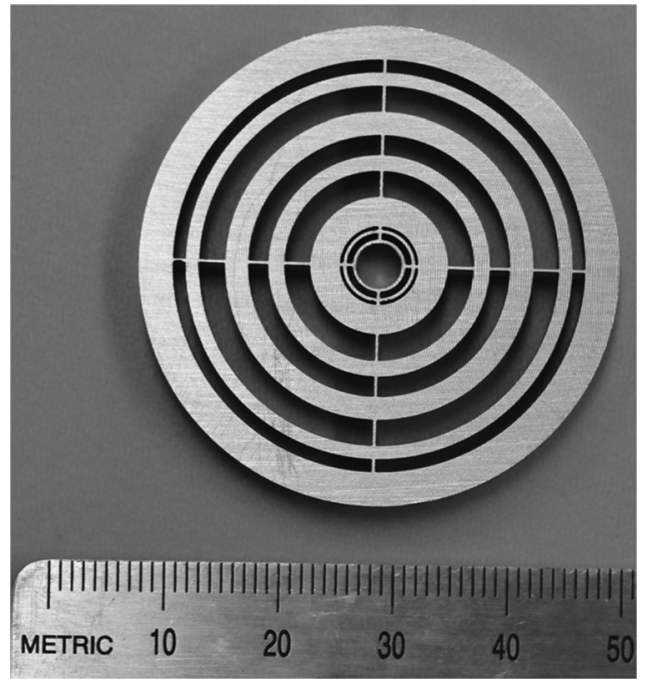

FIG. 2. Photograph of the fabricated acoustic lens. Scale is in millimeters.

entire three-dimensional (3D) structure. Because of the established parameters of radial symmetry for the design, the $2 \mathrm{D}$ model is examined at the maximum cross-sectional diameter at the center of the lens, and this is assumed to be the area of greatest focusing. A photograph of the lens is shown in Fig. 2.

The aim of the theoretical and computational model is to determine the distribution of the acoustic pressure ahead of the structure, and to optimize the dimensions of the structure to obtain highest possible pressure with minimum focal spot diameter. For this purpose it is necessary to solve for the pressure in Eq. (1),

$$
\begin{aligned}
& \nabla^{2} P+k^{2} P=0, \\
& k=\frac{\omega}{c}
\end{aligned}
$$

with the boundary conditions,

$$
\boldsymbol{n} \cdot \nabla P_{1}=\boldsymbol{n} \cdot \nabla P_{2}
$$

where $P$ is the pressure and $c$ is the velocity of the longitudinal acoustic waves, $\omega=2 \pi f, f$ is the frequency of the acoustic waves, $n$ is the unit vector normal to the acoustic field and $\nabla P_{1}$ and $\nabla P_{2}$ are the pressure gradients on each side of the boundary. In addition to the boundary conditions, two other assumptions are made: (1) the wavelength of sound in the solid structure is very large compared to the thickness of the structure at any location and (2) the stiffness of the solid structure is infinitely large compared to the fluid.

The solution for the acoustic pressure fields can be obtained from many different approaches such as finite element method or boundary element method. The complexities of the solution of Eq. (1) are not only in determining the pressure at all locations ahead of the structure, but also in optimizing of the dimensions of the structure. To perform both the computation and the optimization efficiently with high accuracy COMSOL Multiphysics was used in conjunction with a custom hybrid algorithm created by splicing two existing optimization algorithms together.

Due to the complexity associated with the optimization of acoustic lens design, the solution space is highly dimensional. Compared to more traditional optimization methods, such as gradient descent, genetic algorithms afford more flexibility to consider nonlocal candidate solutions. Genetic algorithms (GAs) are designed to find an optimum set of values or features, called genes. The genes used in the optimizing algorithm were represented by the materials state as a binary value of void (air) or solid (aluminum) in the lens structure. The diametric cross section of the lens was divided in to 127 rectangular slices of void and solid. Although the through-thickness of each slice was designated with a binary value, the through-thickness of each slice could be varied in the optimization algorithm. However, using this binary simplification greatly increases the manufacturability of the optimized lens. The collection of all genes after optimization forms a linear lens and by rotating the structure about the axis of symmetry the 3D lens structure was generated.

Genetic algorithms typically begin with two or more seeds, or randomly generated arrays of genes. The algorithm then splices, or mates, these seeds to generate a series of offspring. The quality of these offspring is evaluated by defining a fitness function to determine the best offspring, which are assigned as the parents in the following iteration. The process of breeding parents is repeated until a stopping criterion is met.

The randomness associated with GAs enables the consideration of multiple paths simultaneously. Multiple paths minimize the probability of premature convergence, as the likelihood that all of the paths will prematurely converge is less likely than the probability that one path will prematurely converge. While the inherent randomness may seem haphazard, genetic algorithms enable wide-scope optimization of problems that may not be realistically solvable via the brute force technique. However, it is possible for desirable genes to remain unrepresented by the parents and subsequent offspring. In addition, genetic algorithms are generally ineffective for finding global maximums for highly dimensional problems.

Another type of algorithm that is available is the greedy algorithm. Greedy algorithms (GRs) break complicated problems into a series of smaller problems, or steps. GRs optimize each step independently and combine the smallscale optimizations to estimate the true global maximum. Complex problems, like the work described here, make achieving the global maximum virtually impossible with a GR; however, it is possible to obtain an optimized solution that is sufficiently close to the global maximum. Greedy algorithms are known for their "hill-climbing" capabilities. However, they are innately myopic, and are prone to becoming fixed at a particular local maximum.

Combining GA and GR attributes into a hybrid algorithm, some of the inherent downfalls of the base algorithms are mitigated while capturing the strengths of both (Fig. 3). Initially, the hybrid algorithm (HA) performs like a GA. Numerous seeds are randomly generated to start the algorithm. The HA selects only the single best candidate (based 


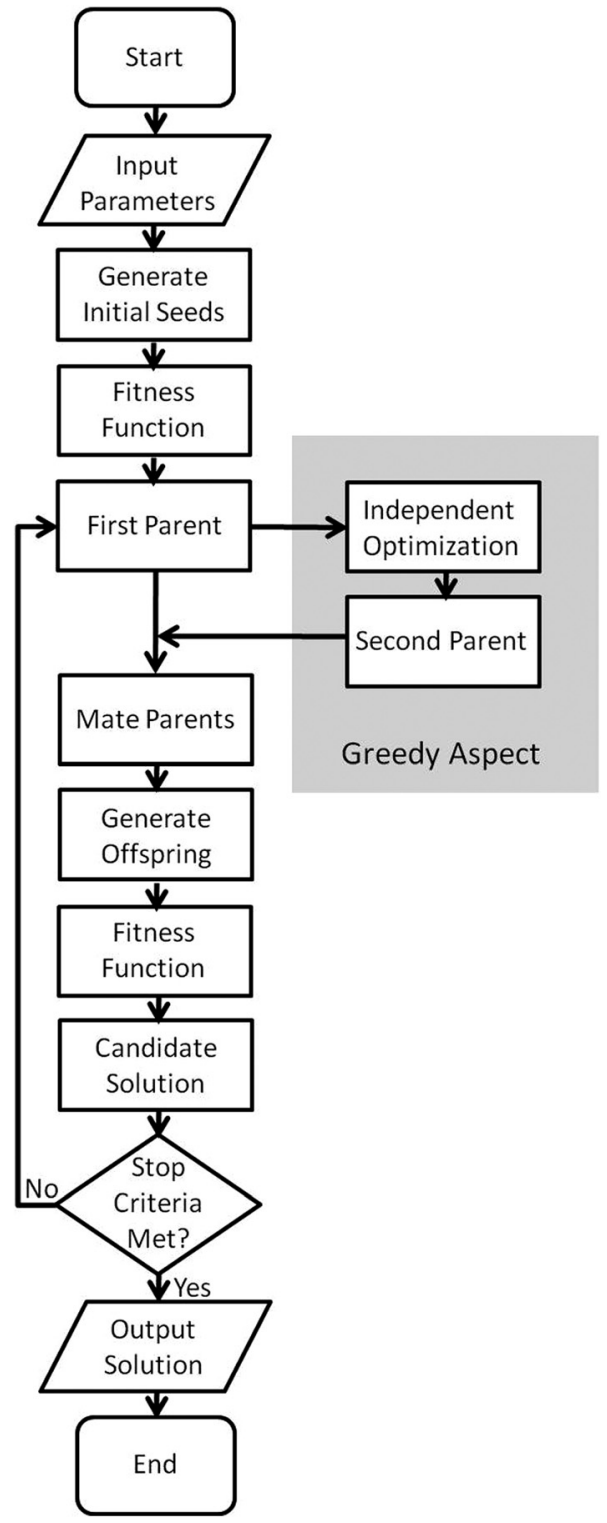

FIG. 3. Flow chart of hybrid algorithm and simulation process to design an acoustic lens.

on the fitness function) to be the first parent (opposed to selecting multiple seeds or offspring to mate in subsequent iterations). The GR aspect of the HA now takes over to generate a second parent by independently switching the state (due to the binary simplification of the genes used) of each cell in the first parent, and determining whether the initial or switched value generates the largest fitness value. This process is repeated for each cell individually to generate a second parent that includes the fittest state from every cell of the first parent. This essentially finds the locally optimum direction that increases the fitness value the most. The GA aspect of the hybrid algorithm resumes after the second parent is generated and the two parents are mated to generate a new set of offspring. The offspring with the largest fitness value is selected as the new first parent and the process is repeated until a stopping criterion is met.

It is important to note two things regarding this algorithm: (1) the algorithm makes no real attempt to find the global maximum, which would be unrealistic for a problem of this complexity, and (2) the goal of generating the second parent by a GR is to introduce genes with desirable characteristics; the second parent is not a candidate solution. In complex problems, the fitness value for the second parent may be insignificant due to neglecting intercell dependencies and introducing competing optimization paths. By mating the parents through the GA aspect of the algorithm offspring with a portion of these new desirable genes are generated. The hybrid nature of this algorithm effectively takes advantage of the GA's ability to randomly search a larger portion of the solution space while maintaining the GR's ability to converge to a local maximum and introduce new genes.

The HA is implemented in MATLAB and continually changes the geometry parameters according to the iteration of the algorithm and then sends the parameters to COMSOL. The structure is then meshed and the pressure field is solved for over the entire domain for a time harmonic case by solving Eq. (5) for the two-dimensional case. Once the pressure field is found, the solution at the position of the focal point is returned from the COMSOL script. The focal point pressure is the fitness (objective) function. The genetic algorithm uses this solution as a comparison measure for each of the geometry cases, and the best is used as parents for the next generation.

The simulation of the acoustic structure is designed to focus $100 \mathrm{kHz}$ incident acoustic waves to a point. This results in a 3D circular ring structure with an overall diameter of $40 \mathrm{~mm}$. The focal length of the simulated lens structure is $6.7 \mathrm{~mm}$ and the focal spot diameter of the highest acoustic pressure is $1.7 \mathrm{~mm}$ in diameter.

\section{EXPERIMENT METHODS AND RESULTS}

A CAD drawing was generated from the model by revolving the $2 \mathrm{D}$ structure about the vertical centerline and adding four webs, one at each quadrant, to hold the rings together thereby making a 3D structure. The lens was manufactured from aluminum using wire electrical discharge machining (EDM) with a minimum feature size of $1 \mathrm{~mm}$ in width. An optical photograph of the acoustic structure is shown in Fig. 2. To characterize the focusing effect of the lens a commercial air coupled transducer with a nominal center frequency of $100 \mathrm{kHz}$ was used as an excitation source. Although the manufacturer quoted center frequency was $100 \mathrm{kHz}$, measurement of displacements on the face of the transducer with a scanning laser vibrometer showed that the transducer had a center frequency of $85 \mathrm{kHz}$ and a bandwidth from $80-90 \mathrm{kHz}$. The transducer was placed at a distance of $80 \mathrm{~mm}$ from lens so that the lens is in the far field region of the transducer. At this distance from the transducer plane waves are expected to be incident on the lens structure. A swept frequency audio source was used to excite the transducer from $80-90 \mathrm{kHz}$.

The diameter of the focal spot determined from the simulation is expected to be $1.7 \mathrm{~mm}$ in diameter. Traditional experimental techniques have severe limitations in characterizing a focal spot and spatial resolution smaller than $1.7 \mathrm{~mm}$ in air. In conventional techniques, a transducer 
of diameter smaller than the spatial resolution would be scanned in front of an acoustic lens. The received signal amplitude is detected and mapped. The $3 \mathrm{~dB}$ width of the highest amplitude would be used to determine the focal spot size. However, for the present application a suitable transducer capable of operating in air in the frequency range of $80-90 \mathrm{kHz}$ is not commercially available. Another common method used for acoustic field distribution characterization is to measure the energy returned to the transmitting transducer from a small spherical reflector. Ideally, the reflector should be smaller than the expected focal spot size, $1.7 \mathrm{~mm}$ in this case. While it is possible to make a reflector of $1 \mathrm{~mm}$ diameter or less, the amount of energy reflecting back to the transducer is found to be within the noise of the transducer. Theoretically, advanced signal processing techniques could be used to isolate this return signal. However, the reflected signal would have to pass through the lens a second time and it is impossible to distinguish between these two independent variations and isolate the effects of each due to their independent nature. Since the traditional methods have severe limitations to characterize spatial resolution smaller than $1.7 \mathrm{~mm}$ in air, two new approaches were developed.

In the first experimental method a circular aperture with a thin elastic membrane stretched over it is placed in front of the lens. The out-of-plane displacements of the membrane during excitation of the acoustic lens are measured with a scanning laser vibrometer. This image of the membrane displacement demonstrated a clear increase in the central region and the focal spot was observed with satisfactory signal to noise ratio. However, the measurements of focal spot distance from the lens face, and the diameter of the focal spot were observed to be different from the results of the simulation. A possible cause for the difference between the experimental measurement and the simulation is the elastic stiffness of the membrane. The elastic nature of the membrane is effectively averaging the incident pressure pulse over a larger area giving the appearance of a much larger region of interaction.

To overcome the limitations of measuring focal spot size by measuring out-of-plane displacements of an elastic membrane, a new approach was developed (Fig. 4). In this method a small $0.35 \mathrm{~mm}$ diameter disk of metalized polymer film is affixed to a $0.05 \mathrm{~mm}$ diameter fiber. The fiber with the metalized disk is stretched diametrically across a $50 \mathrm{~mm}$ circular aperture. The circular aperture with fiber-disk is placed in front of the acoustic lens structure (Fig. 5). The scanning laser interferometer is focused on the metalized

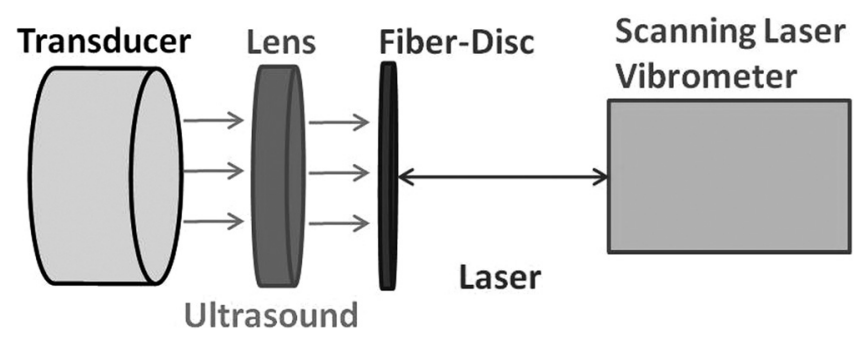

FIG. 4. Block diagram of the excitation of the acoustic lens and measurement of the displacements of the fiber-disk with scanning laser vibrometer.

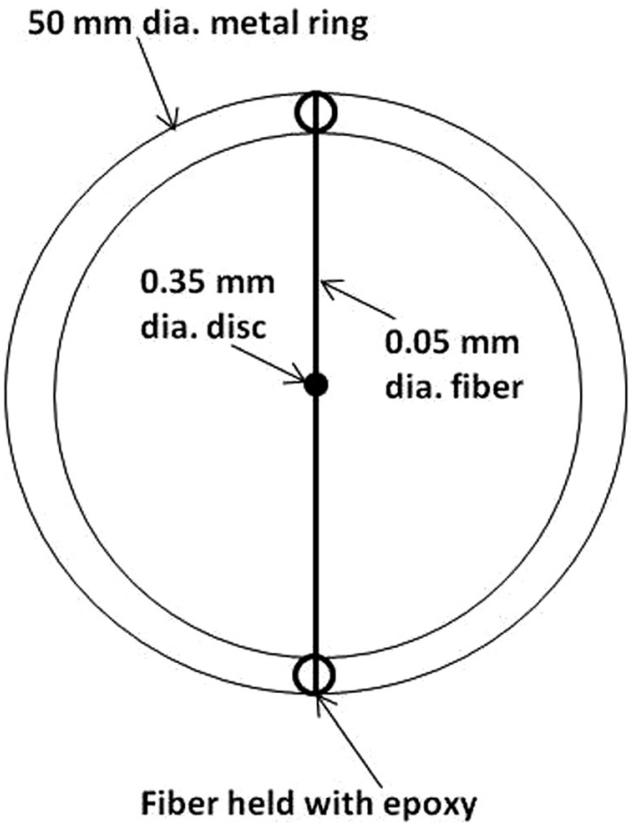

FIG. 5. Schematic of fiber, disk and ring arrangement used in measurement of the focal spot of the acoustic lens. Not to scale.

disk and the out-of-plane displacement is measured when the acoustic transducer is excited. Using manual micrometer stages the aperture is moved from left to right across the lens at varying distances from the lens. This enables the distance from the lens of the maximum focal spot to be determined as well as the width of the focal spot. The scanning vibrometer being used collected out of plane displacements over a frequency range by sweeping the frequency of excitation. Out of plane displacement data from a scanning laser vibrometer is collected over with fiber-reflector arrangement at varying distances from 0.5 to $4 \mathrm{~mm}$ from the lens to determine the optimum focal position of the lens (Figs. 6 and 7). The displacement measurements were repeated seven times to examine the repeatability in measured out of plane displacement. The displacement at each location is converted into acoustic pressure and shown in Fig. 8. The error bars in Fig. 8 at each $\mathrm{x}$-position represent the standard deviation of the average of seven individual measurements. It was observed from Fig. 8 that the acoustic lens structure operated most efficiently in air in the frequency range of $80-90 \mathrm{kHz}$. The maximum acoustic pressure was located at a distance of 2.5 $\mathrm{mm}$ from the surface of the lens. The average diameter of the focal spot, full width at half maximum, was determined to be $3.5 \pm 0.12 \mathrm{~mm}$ for the bandwidth of the excitation transducer, 80-90 kHz (Fig. 8). Assuming $343 \mathrm{~m} / \mathrm{s}$ for the velocity of sound in air at $20{ }^{\circ} \mathrm{C}$ and $85 \mathrm{kHz}$ for the operating frequency of the acoustic lens, the average diameter of the focal spot was determined to be 0.88 of the incident wavelength $(4.0 \mathrm{~mm})$ of sound in air.

The computationally simulated acoustic field for the aperiodic flat lens structure was optimized to operate at 100 $\mathrm{kHz}$. The predicted focal length is $6.7 \mathrm{~mm}$ and the spatial resolution is $1.7 \mathrm{~mm}$. Although the dimensions of the lens were optimized for $100 \mathrm{kHz}$, when the lens is manufactured its best performance occurs in the frequency range of 80-90 


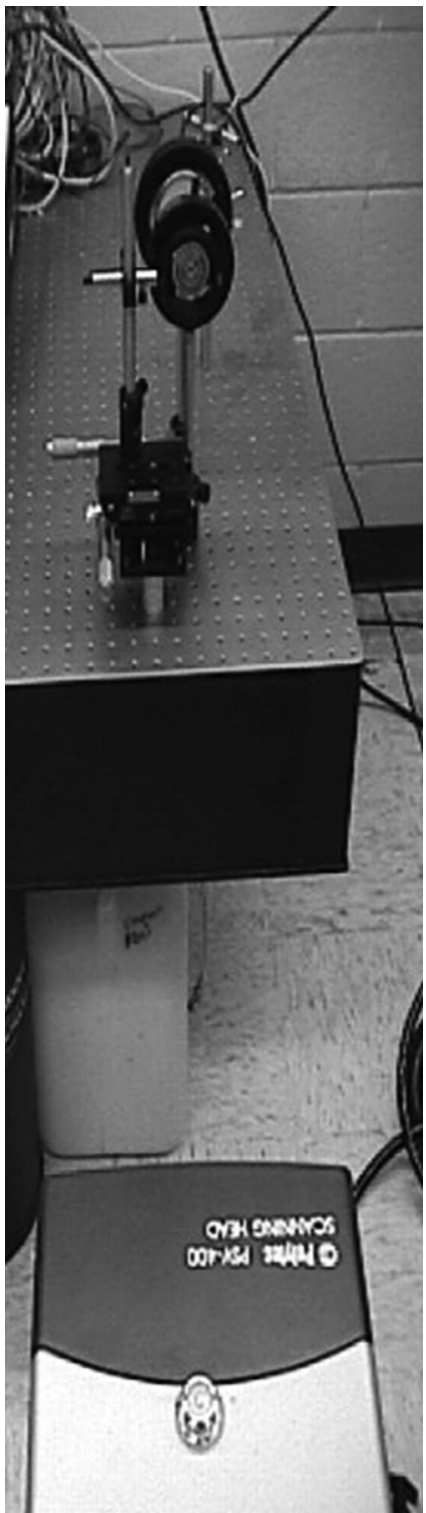

FIG. 6. Photograph of the test up illustrating the position of the scanning laser vibrometer (bottom of photo), fiber-disk, lens and transducer (top).

$\mathrm{kHz}$, which was the bandwidth of the air transducer. The experimentally determined focal length is $2.5 \mathrm{~mm}$ and the spatial resolution is $3.5 \mathrm{~mm}$.

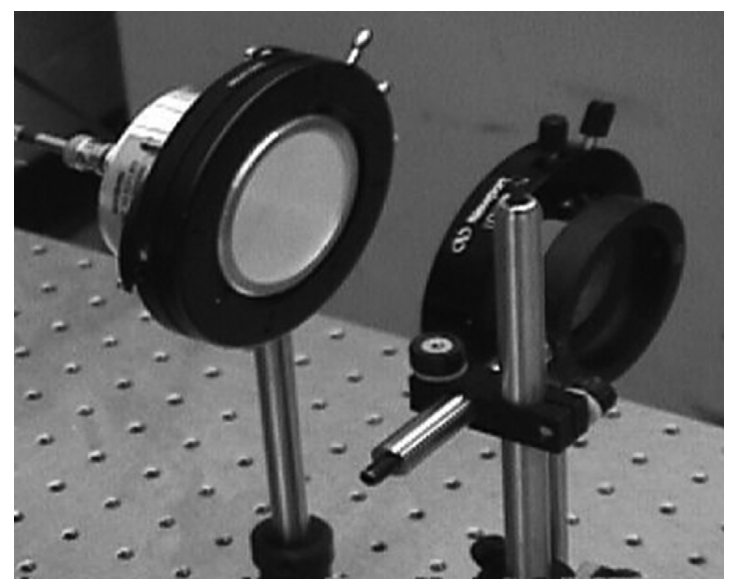

FIG. 7. Photograph of transducer (left), lens, and fiber-disk (right)

\section{DISCUSSION}

Computer modeling and simulation approaches, combined with the hybrid genetic algorithm used in the paper, show that a structure can be designed to produce a flat acoustic lens. The flat acoustic lens structure manufactured based on dimensions generated by computer model was experimentally evaluated. The manufactured lens, although optimized by computer modeling and simulation to operate at $100 \mathrm{kHz}$, operates most effectively in the frequency range of 80-90 kHz. Experimentally determined focal length and the spatial resolution differ from the computer simulation by $63 \%$ and $51 \%$, respectively. A number of factors could cause the observed deviations of the experimental behavior from the simulation.

The most likely factors for the observed deviations are related to the excitation transducer and manufacturing of the lens. The excitation transducer used is nominally centered at $100 \mathrm{kHz}$. Characterization of the transducer with the scanning laser vibrometer showed that it has a center frequency of $85 \mathrm{kHz}$ with a bandwidth of $10 \mathrm{kHz}$. The computer simulation, however, assumes a perfect $100 \mathrm{kHz}$ source. Characterization of the excitation transducer to determine both the frequency and bandwidth, which could then be input into a computer simulation, is needed to yield more accurate results from the simulation.

Although the design parameters obtained for fabricating the lens from the computer simulation are extremely precise, the tolerances during manufacturing could only be controlled to $\pm 0.03 \mathrm{~mm}$. This affects the dimension of the rings, and in particular the thinnest rings. The thinnest rings could deviate up to $5 \%$ from the computer simulated design, which could have a significant impact on the performance characteristics of the lens. Furthermore, the computer simulation used a 2D approximation that results in 3D structure of concentric rings. During fabrication, webs are added to hold the rings together. The resulting increase in the complexity of the acoustic field distribution is an additional source of error. It should be noted that for a periodic grating focusing in the far field, due to scattering from the central region of the lens structure is larger than the error due to scattering from the outer regions. Revolving a $2 \mathrm{D}$ periodic grating to generate a $3 \mathrm{D}$ structure would compound the error for this case. The role of this error for near field gratings, of the type described in this paper, is not well established. The combination of the various effects may significantly affect the frequency, focal length and the spatial resolution of the lens structure when compared to theoretical prediction.

While fabrication can alter the operating parameters of the lens, measurement of acoustic field distribution in air to determine spatial resolution of the lens is also challenging and can introduce uncertainties of its own. The fiber-disk arrangement is an innovative method to map the acoustic field distribution in air. The displacements of the fiber-disk depend on the elastic properties of both the fiber and the disk, and the tension in the fiber. The ideal situation to determine the spatial resolution is for the transducer, the lens, and the fiber-disk arrangement to be aligned coaxially. Small variations in this alignment will introduce errors particularly 


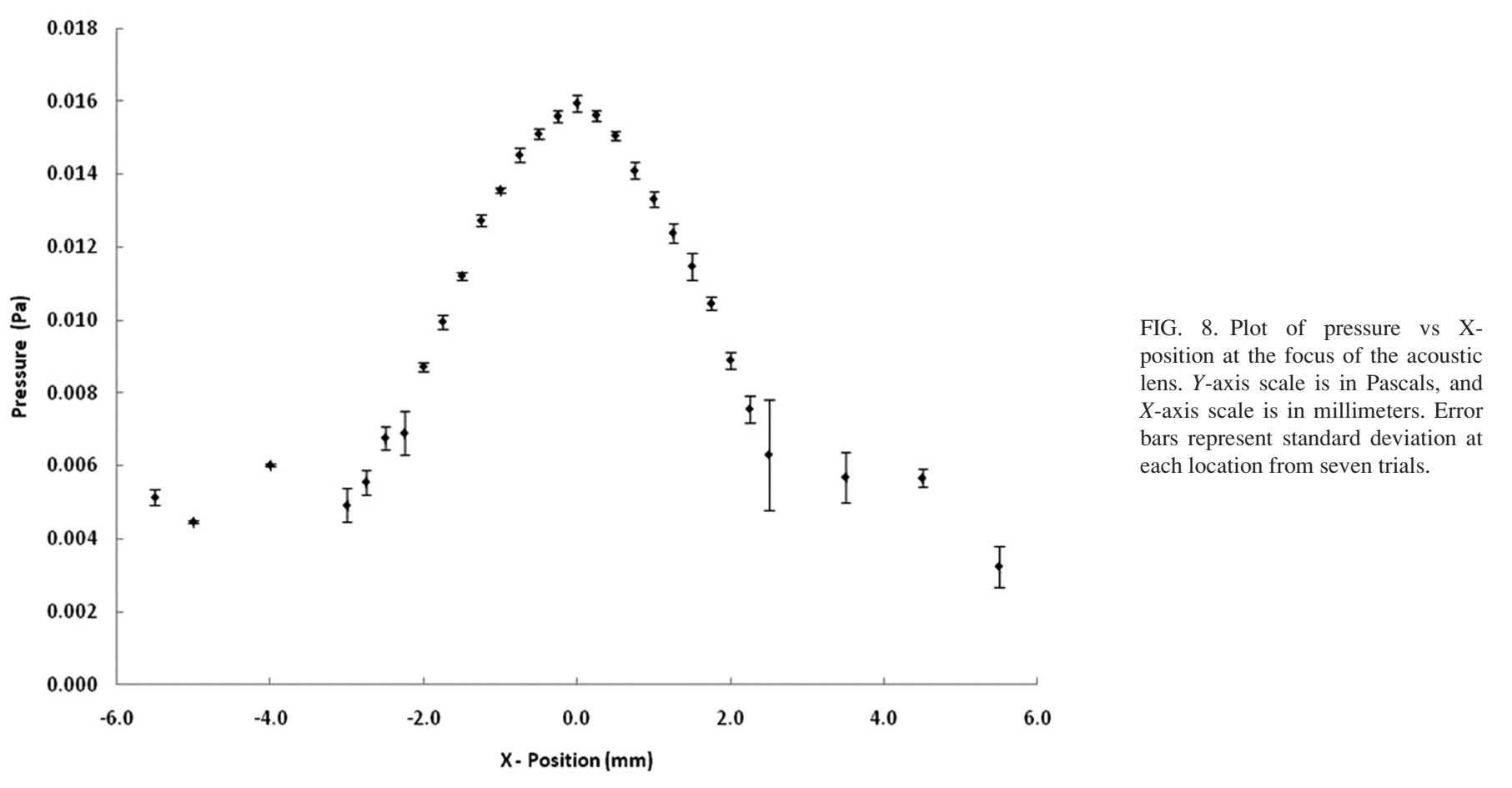

in the determination of both focal length and spatial resolution. Nonparallel arrangements can produce decreased spatial resolution compared with the theoretical calculations.

Although the computational modeling is very successful in providing an avenue to develop flat acoustic structure, significant improvements are needed to enhance its predictive capabilities. Currently, the model uses a relationship to compute pressure at every point from an incident plane wave interacting with the structure. It does not take into account possible resonances of the individual elements of the lens structure, or any bandwidth in the incident wave. Adding these inputs to the model would increase its fidelity and make it a more robust tool for acoustic lens design.

\section{v. CONCLUSIONS}

An acoustic lens with an aperiodic structure intended to operate in air was designed with the help of computational simulations using a hybrid genetic-greedy algorithm. The hybrid genetic-greedy algorithm works effectively to design the lens by minimizing the number of lens elements and reducing computation time. The computational simulation allows optimization of the dimensions of the individual elements of the lens along with the acoustic field distribution. The lens design was optimized to operate at $100 \mathrm{kHz}$ with a focal length of $6.7 \mathrm{~mm}$ and spatial resolution of 1.7 $\mathrm{mm}$. The approach of computer simulation minimizes the need to test multiple configurations of the lens. The output lens element dimensions from the computer simulation were used to fabricate a flat acoustic lens structure. An air coupled acoustic transducer was used to propagate acoustic waves towards the flat acoustic lens. Characterization of acoustic field distribution of the lens, an area less than $4 \mathrm{~mm}^{2}$, was found to be challenging. An innovative experimental technique using a $0.35 \mathrm{~mm}$ diameter metal coated polymer disk attached via a $0.05 \mathrm{~mm}$ fiber to a 50 $\mathrm{mm}$ diameter ring was developed to measure the acoustic field of the lens with a laser interferometer. The lens was found to operate efficiently in the frequency range of 80-90 $\mathrm{kHz}$, which was the bandwidth of the excitation transducer, although the lens was optimized to operate at $100 \mathrm{kHz}$. The focal length was found to be $2.5 \mathrm{~mm}$ instead of $6.7 \mathrm{~mm}$ and the spatial resolution was determined to be $3.5 \mathrm{~mm}$ compared with $1.7 \mathrm{~mm}$ from simulation. Possible reasons for the difference between the experimental measurement and the computer simulations were analyzed and attributed to non-optimal frequency of the excitation transducer, fabrication tolerances, and uncertainties in the experimental arrangements and instrumentation. Possible improvements in the computational simulation include implementing a 3D simulation of the lens and optimizing for the focal point with the best combination of resolution and pressure.

\section{ACKNOWLEDGMENTS}

The authors would like to acknowledge Ed Klosterman, Rick Reibel, Julie Piddock, and Brooke Johnson for their assistance in this work. Portions of this work were performed under U.S. Air Force contracts FA8650-09-D-5224 and FA8650-09-2-5800. Authors would like to thank the anonymous reviewers for their critical comments that provided additional insight into the discussion of the results.

\footnotetext{
${ }^{1}$ A. Briggs and O. Kolosov, Acoustic Microscopy (Oxford University Press, New York, 2009).

${ }^{2}$ M. Fortunko, W. P. Dube, and J. D. McColskey, "Gas-coupled acoustic microscopy in the pulse-echo mode," Proc. IEEE Ultrasonics Symp. 2, 667-671 (1993).

${ }^{3}$ M.C. Bhardwaj, "Evolution of piezoelectric transducers to full scale noncontact ultrasonic analysis mode," World Conference on Non-Destructive
} 
Testing-2004, Montreal, Canada, http://www.ultrangroup.com/Company/ Publications/Default.aspx (Last viewed 6/2011).

${ }^{4} \mathrm{M}$. Born and E. Wolf, Principles of Optics: Electromagnetic Theory Of Propagation, Interference and Diffraction Of Light, 6th ed. (Pergamon, New York, 1991).

${ }^{5}$ B. Hadimioglu, E. G. Rawson, R. Lujan, M. Lim, J. C. Zesch, B. T. KhuriYakub, and C. F. Quate, "High-efficiency Fresnel acoustic lenses," Proc. IEEE Ultrasonics Symp. 1, 579-582 (1993).

${ }^{6}$ J. B. Pendry, "Negative refraction makes a perfect lens," Phys. Rev. Lett. 85, 3966-3969 (2000).

${ }^{7}$ N. Fang, D. Xi, J. Xu, M. Ambati, W. Srituravanich, C. Sun, and X. Zhang, "Ultrasonic metamaterials with negative modulus," Nature Mater. 5, 452-456 (2006).

${ }^{8}$ S. Guenneau, A. Movchan, G. Petursson, and S. A. Ramakrishna, "Acoustic metamaterials for sound focusing and confinement," New J. Phys 9, 399 (2007).

${ }^{9}$ S. Zhang, L. Yin, and N. Fang, "Focusing ultrasound with an acoustic metamaterial network," Phys. Rev. Lett. 102, 194301 (2009).

${ }^{10}$ S. H. Lee, C. M. Park, Y. M. Seo, Z. G. Wang, and C. K. Kim, "Acoustic metamaterial with negative modulus," J. Phys.: Condens. Matter 21, 175704 (2009).

${ }^{11}$ H. H. Huang and C. T. Sun, "Wave attenuation mechanism in acoustic metamaterial with negative effective mass density," New J. Phys. 11, 013003 (2009).

${ }^{12}$ C. Qiu, X. Zhang, and Z. Liu, "Far-field imaging of acoustic waves by a two-dimensional sonic crystal," Phys. Rev. B 71, 054302 (2005).

${ }^{13}$ L. Feng, X.-P. Liu, Y.-B. Chen, Z.-P. Huang, Y.-W. Mao, Y.-F. Chen, J. $\mathrm{Zi}$, and Y.-Y. Zhu, "Negative refraction of acoustic waves in twodimensional sonic crystals," Phys. Rev. B 72, 033108 (2005).
${ }^{14}$ F. Cai, F. Liu, Z. He, and Z. Liu, "High refractive-index sonic material based on periodic subwavelength structure," Appl. Phys. Lett. 91, 203515 (2007).

${ }^{15} \mathrm{X}$. Ao and C. T. Chan, "Far-field image magnification for acoustic waves using anisotropic acoustic metamaterials," Phys. Rev. E 77, 025601(R) (2008).

${ }^{16}$ S. Thongrattansiri and V.A. Podolskiy, "Hypergratings: nanophotonics in planar anisotropic metamaterials," Opt. Lett. 34, 890-892 (2009).

${ }^{17}$ A. Håkansson, F. Cervera, and J. Sánchez-Dehesa, "Sound focusing by flat acoustic lenses without negative refraction," Appl. Phys. Lett. 86, 054102 (2005).

${ }^{18}$ D. Torrent and J. Sánchez-Dehesa, "Acoustic metamaterials for twodimensional sonic devices,” New J. Phys. 9, 323 (2007).

${ }^{19}$ A. Grbic, L. Jiang, and R. Merlin, "Near-field plates: subdiffraction focusing with pattered surfaces," Science 320, 511-513 (2008).

${ }^{20}$ M. F. Imani and A. Grbic, "Near field focusing with a corrugated surface," IEEE Antennas Wireless Propag. Lett. 8, 421-424 (2009).

${ }^{21}$ H. Chen, L. Ran, D. Wang, J. Huangfu, Q. Jiang, and J. A. Kong, "Metamaterial with randomized patterns for negative refraction of electromagnetic waves," Appl. Phys. Lett. 88, 031908 (2006).

${ }^{22}$ L. Sanchis, A. Yánez, P. L. Galindo, J. Pizarro, and J. M. Pastor, "Threedimensional acoustic lenses with axial symmetry," Appl. Phys. Lett. 97, 054103 (2010).

${ }^{23}$ E. Twerdoski, M. Von Buttlar, A. Habib, R. Wannemacher, and W. Grill, "Acoustic holographic imaging by scanning point contact excitation and detection in piezoelectric materials," 9th European Conference on NDT, September 2006, Berlin, Germany, http://www.ndt.net/article/ecndt2006/ doc/Fr.1.8.4.pdf (Last viewed 6/2011). 